\title{
Effect of povidone iodine, chlorhexidine digluconate and toyocamycin on amphizoic amoebic strains, infectious agents of Acanthamoeba keratitis - a growing threat to human health worldwide
}

\author{
Marcin Padzik ${ }^{1, A-F} \oplus$, Wanda Baltaza ${ }^{1, B-D \oplus}$, David Bruce Conn ${ }^{2,3, E-F} \oplus$, Jacek P. Szaflik ${ }^{4, E-F} \oplus$,

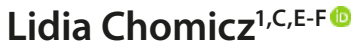 \\ ${ }^{1}$ Department of Medical Biology, Medical University, Warsaw, Poland \\ 2 Department of Invertebrate Zoology, Museum of Comparative Zoology, Harvard University, Cambridge, USA \\ ${ }^{3}$ One Health Center, Berry College, School of Mathematical and Natural Sciences, Mount Berry, Georgia, USA \\ ${ }^{4}$ Department of Ophthalmology, SPKSO Ophthalmic Hospital, Medical University, Warsaw, Poland \\ A - Research concept and design, B - Collection and/or assembly of data, C - Data analysis and interpretation, \\ $D$ - Writing the article, E - Critical revision of the article, F - Final approval of article
}

\begin{abstract}
Padzik M, Baltaza W, Conn DB, Szaflik JP, Chomicz L. Effect of povidone iodine, chlorhexidine digluconate and toyocamycin on amphizoic amoebic strains, infectious agents of Acanthamoeba keratitis - a growing threat to human health worldwide. Ann Agric Environ Med. 2018; 25(4): 725-731. doi: 10.26444/aaem/99683
\end{abstract}

\begin{abstract}
Introduction. Free-living amoebae, ubiquitous in outer environments, in predisposing circumstances may exist as parasites, infectious agents of Acanthamoeba keratitis. In recent decades, the vision-threatening corneal infection is a growing human health threat worldwide, including Poland. The applied therapy is often ineffective due to diagnostic mistakes, various pathogenicity of Acanthamoeba strains and high resistance of cysts to drugs; many agents with possible anti-amoebic activity are still being tested. In the presented study, selected chemicals are investigated in terms of their in vitro effect on corneal and environmental Acanthamoeba strains.

Materials and method. Samples of a corneal isolate from a patient with severe Acanthamoeba keratitis,of assessed on the basis of genotype associations of 18S rRNA and the type strain, Acanthamoeba castellanii Neff cultivated in bacteria-free condition, were exposed to povidone iodine, chlorhexidine digluconate or toyocamycin. In vitro population dynamics of the strains were monitored and compared to those of control cultures.

Results. All chemicals showed anti-amoebic effects with different degrees of effectiveness. Significant differences were observed in the in vitro population dynamics, and the morpho-physiological status of A. castellanii Neff T4 and corneal strains determined as A. polyphaga T4 genotype, exposed to povidone iodine or toyocamycin, in comparison with chlorhexidine taken as reference.

Conclusions. Time-dependent amoebstatic in vitro effects were demonstrated for all agents, in particular, the results of assays with povidone iodine are promising. No significant stimulation of encystation appeared; however, as cysticidal efficacy of chemicals is expected, complementary research is needed on different Acanthamoeba strains with modified agent concentrations and method application.
\end{abstract}

\section{Key words}

in vitro, Acanthamoeba Keratitis, A. polyphaga, T4 genotype, A. castellanii Neff, povidone iodine, chlorhexidine digluconate, toyocamycin effects

\section{INTRODUCTION}

Free-living amoebae (FLA) belonging to the genus Acanthamoeba are exozoic organisms widely distributed in various parts of the world, including Poland [1-16]. The protists exist as active feeding trophozoite forms that under adverse conditions transform into double-walled dormant cysts. The amoebae complete their life cycles in outer natural and man-made environments. They occur in various soil and aquatic habitats: in the soil, air, water (including tap and chlorinated water), drinking water systems, bottled mineral

Address for correspondence: Marcin Padzik, Department of Medical Biology Medical University of Warsaw, Warsaw, Poland, Nowogrodzka 73, 02-018 Warszawa, Poland marcin.padzik@wum.edu.pl

Received: 20.09.2018; accepted: 10.11.2018; first published: 03.12.2018 water, thermal recreational waters, and swimming pools; they are also often isolated from vegetables and fruits. The protists are found in dust and sewage, in air-conditioning systems, and humidifiers; they are also detected in health facilities, on surfaces of equipment, contact lenses and lens boxes, on accessories, surgical instruments, in dialyzers and in dental irrigation units. Moreover, the amoebae may also act as vehicles/vectors for fungi, protists, viruses and/ or bacteria, including species pathogenic for humans from the genera Escherichia, Chlamydia, Pseudomonas, Legionella, and Candida, which may survive and even proliferate within the Acanthamoeba trophozoites $[15,17,18]$.

In favourable conditions, some species of the amoebae may enter and colonize various animals, including humans, and exist as endozoic organisms - facultative parasites [4, 
6, 9-18]. For this reason, the FLA are considered to be amphizoic protists because they are able to exist in two different modes: as free-living exozoic amoebae and parasitic endozoic organisms causing pathogenic effects.

Some Acanthamoeba species may cause serious diseases in humans: rare but life-threatening granulomatous amoebic encephalitis, an opportunistic disease that develops in immuno-compromised individuals $[2,3,4,17,19]$, and the non-opportunistic, progressive, sight-threatening eye disease, Acanthamoebic keratitis (AK) in immuno-competent patients $[4,15,16,17,20,21,22,23]$.

The amoebic infection of the human cornea was first reported by Nagington et al. [20]. Disease symptoms include tearing, redness, severe pain, photophobia, visual impairment, corneal ulcerations, and epithelial defects. Up to $90 \%$ of AK cases have been reported in contact lens wearers and related to improper contact lens hygiene. It is emphasized in the literature, and also confirmed by the experience of the authors of the current study, that $\mathrm{AK}$ is diagnosed with increasing frequency, including in Poland, along with the spread of the use of contact lenses $[8,15,16$, 20-29]. Important risk factors predisposing to Acanthamoeba infections in humans, apart from wearing contact lenses, are damage to the corneal epithelial cells, eye surgery, and exposure of the eye to water containing amphizoic amoeba trophozoites and/or cysts [15, 17, 21, 28, 30].

In recent decades, vision-threatening AK corneal infection has become a growing threat to human health worldwide. Applied therapy is often disappointing due to misdiagnostics, different pathogenicity of Acanthamoeba species and extreme resistance of Acanthamoeba cysts to disinfectants and drugs $[8,15,16,17,23,30,31,32]$. For this reason, during the years of multicentre research, conducted also by the authors of the current study, different agents with possible activity against various Acanthamoeba strains are still being tested.

\section{OBJECTIVE}

In the current research, in vitro effects of selected chemicals were investigated on pathogenic Acanthamoeba isolate acquired and identified from complicated infectious keratitis, and on the environmental strain. Influence of the agents on the amoebic population dynamics in vitro was also examined, compared and evaluated. The study was performed in accordance with the tenets of the Declaration of Helsinki.

\section{MATERIALS AND METHOD}

Acanthamoeba strains investigated. The strains included in this study were two different Acanthamoeba species, environmental and pathogenic, cultivated in vitro in the laboratory of the Department of Medical Biology, Medical University of Warsaw, Poland.

The material of the ocular isolate was derived from a nonwearer of contact lenses with a history of swimming in a lake, referred to hospital from another centre following previous misdiagnosis and unsuccessful treatment with antibacterial and antifungal medications.

Clinically, corneal ulcer and active epithelial inflammation in the course of complicated, severe Acanthamoeba keratitis were detected by non-invasive methods: the slit-lamp and in vivo confocal microscopy.

The material acquired from corneal scrapings was examined with a light microscope to visualize cysts or/and trophozoites of the amoebae. Samples of the corneal strain were cultured in vitro in sterile $15.0 \mathrm{ml}$ tubes. Cultivation of this amoebic strain was performed under bacteria-free conditions in BSC medium (composed of Bacto Casitone, Difco, dissolved in water, enriched with $10 \%$ calf serum from Wytwórnia Surowic i Szczepionek, Lublin, with the addition of an aqueous solution of antibiotics: streptomycinum, penicilinum), as in earlier research $[19,23,27,28]$, at $26^{\circ} \mathrm{C}$ and regularly sub-cultured twice a month.

Specific identification of the corneal strain was performed using molecular techniques based on genotype associations of the 18S rRNA gene sequence. An Acanthamoeba specific PCR following the protocol established by Schroeder et al. [33] was applied. Direct sequencing of the obtained PCR product was performed. The sequence was analyzed using GeneStudio Pro Software, and compared with data from the GenBank to establish the genotype of the isolate.

The environmental type strain A. castellanii Neff (classified earlier as a species belonging to Acanthamoeba group II), defined by Schroeder et al. [33] for ATCC 30010 A. castellanii Neff T4 genotype, cultivated and monitored for years in the same BSC growth medium in the Department of Medical Biology laboratory, Medical University of Warsaw, Poland.

Acanthamoeba populations investigated were sub-cultured twice a month and their samples regularly observed for in vitro growth under direct light microscope. A range of amoeba number of two or three counts with the use of Bürker's hemocytometer calculated for $1 \mathrm{ml}$ of culture medium was assessed. Before the exposure to chemicals, the dynamics of developmental forms of the amoebic strains was monitored during each sub-culturing and in the exponential growth phase.

Chemicals tested for effects on Acanthamoeba strains. Three compounds were tested for their potential in vitro activity against the Acanthamoeba strains investigated: povidone iodine PI, chlorhexidine digluconate CXD, and the adenosine analogue toyocamycin Toyo.

The monitoring of in vitro dynamics of amoebic strains was conducted during each sub-culturing and in subsequent growth phase, at the beginning, in the log phase; before the exposure to chemicals the overall amoebae count was in a range from $2-5 \times 10^{4} / 1 \mathrm{ml}$. Seven days after current subculturing, in the exponential phase of the cultivation cycle, samples of cultures of Acanthamoeba corneal strain and type strain A. castellanii Neff T4 genotype were exposed to the tested chemicals and compared in terms of their in vitro susceptibility to these agents. All experiments were performed in sterile $1.5 \mathrm{ml}$ tubes. $25 \mu$ lof the tested compound were added to the $475 \mu$ l of calibrated culture. Low to mid concentrations of chemicals non- toxic for human cells were applied; thus, inhibitory drug concentrations were not determined.

Agents with confirmed and possible in vitro activity against the Acanthamoeba protozoans were applied.

Povidone Iodine Solutio $10 \%$ Teva (PI) (Teva Pharmaceuticals, Poland), a complex of iodine, the bactericidal component with povidone, disinfectant and preoperative antiseptic was dissolved to a final concentration of $0.5 \%(5 \mathrm{mg} / \mathrm{ml})$. 
Chlorhexidine digluconate (CXD), a standard antiseptic belonging to the biguanide family, was used as the reference drug against Acanthamoeba. 20\% solution (Zakłady Farmaceutyczne Polfa, Łódź, Poland) was dissolved in purified water and a final concentration of $0.02 \%$ was applied in these investigations.

Toyocamycin (Toyo), an adenosine analogue selected for this research as an agent with confirmed anti-protozoan activity, (but not for Acanthamoeba spp.), synthesized according to Sharma et al. [34] was tested (kindly provided by Prof. Zygmunt Kazimierczuk from the Division of Organic and Food Chemistry, Warsaw University of Life Sciences). Working stock solution in a concentration $1000 \mu \mathrm{M} / \mathrm{ml}$ was prepared by dissolving the compound powder in $9.6 \%$ ethanol. The final concentrations of toyocamycin, $25 \mu \mathrm{M} /$ $\mathrm{ml}$ and $50 \mu \mathrm{M} / \mathrm{ml}$ were obtained by dissolving the working stock in purified water. In addition, purified water and $0.48 \%$ ethanol-solvent were added to control cultures containing only amoebae, and subjected to the same procedure used for experimental cultures.

The effect of the compounds on Acanthamoeba strains was assessed for each strain following $24 \mathrm{~h}, 48 \mathrm{~h}, 96 \mathrm{~h}, 120 \mathrm{~h}$ and $144 \mathrm{~h}$ exposure, and compared with the control culture count considered as $100 \%$. The level of statistical significance was set at $\mathrm{p}<0.05$.

The dynamics of amoebic strains were monitored in vitro. A range of amoeba number of two or three counts with the use of Bürker's hemocytometer, calculated for $1 \mathrm{ml}$ of culture medium, was assessed in subsequent days of the exponential growth phase. The percentage of particular stages: trophozoites and cysts, morpho-physiological status of these amoebic forms, and particularly in vitro viability of amoebae from both strain populations, were monitored. All assays were repeated twice or thrice; results were analyzed statistically (ANOVA, Student-Newman-Keuls). Values of $\mathrm{p}$ $<0.05$ were considered significant.

\section{RESULTS}

Examination of the material acquired from corneal scrapings with the use of a light microscope revealed live trophozoites forming acanthopodia characteristic for Acanthamoeba genus, and also double-walled cysts. The environmental Acanthamoeba castellanii Neff strain and the corneal strain included in this investigation monitored in vitro showed low amoeba number in the early adaptive phase of cultivation that successively increased in the log, exponential growth phase.

Specific identification of the corneal isolate performed using molecular techniques based on genotype associations of the 18S rRNA gene sequence, following the protocol established by Schroeder et al. [33], indicated that the causative agent of severe AK course is the Acanthamoeba strain determined as A. polyphaga Page-23; ATCC 30871 representing T4 genotype, similar to the genotype of $A$. castellanii Neff maintained and monitored for years in our laboratory $[27,28,32]$.

There were statistically significant differences in the log growth phase in the density of the protozoan population at $26^{\circ} \mathrm{C}$ : viable amoeba count range of the environmental strain was distinctly higher than the number of the eye isolate, $10-18 \times 10^{4}$ and $1-5 \times 10^{4}$, respectively $(\mathrm{p}<0.05)$. The majority $(95 \%-98 \%)$ were the trophozoite forms.
Effect of chemicals on Acanthamoeba environmental and corneal strains. The chemicals investigated showed anti-amoebic effects with various degrees of effectiveness, although the ethyl alcohol concentration had no significant influence on amoebae in all assays. Differences were observed in the population dynamics of $A$. castellanii Neff strain and A. polyphaga T4 genotype exposed to povidone iodine or toyocamycin in comparison to the chlorhexidine digluconate which at was taken as the reference.

An amoebostatic activity of all agents tested was revealed but it was not connected with intense amoebic encystation expressed as increased statistically significant cyst frequency; none of the compounds in the concentration applied in this study induced this process. The amoebic cyst percentage was variable throughout the duration of the experiment around $0.0-5.2 \%$, because the differences revealed in cultures of particular Acanthamoeba strains were insignificant statistically for all agents applied (not presented in detail).

Comparative assessment of the in vitro cultivated Acanthamoeba strain populations after exposure to chemicals showed changes in morpho-physiological status, number of trophozoites and cysts, as well as in proportion between developmental forms, in comparison to the respective control cultures. The changes were expressed as differences in dynamics and viability of amoebic strains examined after $24 \mathrm{~h}, 48 \mathrm{~h}, 96 \mathrm{~h}, 120 \mathrm{~h}$ and $144 \mathrm{hrs}$ exposure to tested chemicals.

Anti-amoebic effects against $A$. polyphaga T4 genotype were detected after different time exposure to the particular tested agents.

Chlorhexidine digluconate is an antiseptic/disinfectant with a broad spectrum of activity and confirmed effectiveness against Acanthamoehba strains. In the beginning, throughout the 4 days after exposure, a variable influence of the chemical on the corneal strain was observed. A clear, statistically significant amoebostatic effect of the agent was revealed just after $120 \mathrm{~h}$ incubation. CXD is used as a topical drug in AK therapy and for this reason, the results obtained for this chemical were included as the reference, and the effects of povidone iodine and toyocamycin on the amoebae were compared to those revealed for CXD.

There were differences in the dynamics and viability of corneal and environmental amoebic populations examined microscopically after 24, 48, 96, 120 and $144 \mathrm{hrs}$ exposure to particular chemicals. Statistically significant changes in the percentage of viable amoebae in comparison to data regarding cultures not exposed to these chemicals appeared from $48 \mathrm{~h}$ and $96 \mathrm{~h}$ for Toyo and $96 \mathrm{~h}$ for PI, compared to $120 \mathrm{~h}$ for CXD (Tab. 1).

Moreover, microscopic examination of samples of the amoebic cultures revealed that their exposure to the tested chemicals caused changes in the morpho-physiological status of amoebic populations in comparison with that observed in the control cultures. This was expressed not only as a decrease in the general number of amoebae, but also in the sporadic appearance of dividing trophozoites as well as in the frequent occurrence of rounded forms, moving slowly or motionless, without forming acanthopodia.

The clear, statistically significant amoebostatic effect on the eye amoebic strain was revealed in assays with PI. This was reflected as a decrease in the number of live amoebae to $30.8 \%$, observed after 4 days of exposure, in comparison to specimens counted in control cultures that were assumed as $100 \%(\mathrm{p}<0.05)$. A similar level of the number of live 
amoebae $(29.9 \%)$ was achieved after 5 days of exposure in assays with CXD. More reduced number of the amoebae to $16.7 \%$ and $22.2 \%(\mathrm{p}<0.05)$ that may be considered as antiamoebic effect of PI was detected in 5 and 6 days exposure to this agent with cultivated amoebae of the corneal isolate to this agent (Tab. 1).

Table 1. In vitro sensitivity of $A$. polyphaga T4 corneal strain to tested agents

\begin{tabular}{lcccccc}
\hline \multicolumn{6}{c}{ Live amoebae after exposure to the agents* by the following hours: } \\
\hline Agent & $\begin{array}{c}\text { Concen- } \\
\text { tration }\end{array}$ & $24 h$ & $48 \mathrm{~h}$ & $96 \mathrm{~h}$ & $120 \mathrm{~h}$ & $144 \mathrm{~h}$ \\
\hline $\begin{array}{l}\text { Povidone iodine } \\
0.5 \%\end{array}$ & $100 \%$ & $89.9 \%$ & $\underline{30.8 \%}$ & $\underline{16.7 \%}$ & $\underline{22.2 \%}$ \\
\hline $\begin{array}{l}\text { Chlorhexidine. } \\
\text { digluconate }\end{array}$ & $0.02 \%$ & $74 . \%$ & $75 \%$ & $68.2 \%$ & $\underline{29.9 \%}$ & $\underline{28.8 \%}$ \\
\hline Toyocamycin & $25 \mu \mathrm{M} / \mathrm{ml}$ & $117.6 \%$ & $84.4 \%$ & $\underline{47.2 \%}$ & $\underline{53.4 \%}$ & $\underline{37.4 \%}$ \\
\cline { 2 - 7 } & $50 \mu \mathrm{M} / \mathrm{ml}$ & $116.2 \%$ & $\underline{74.3 \%}$ & $\underline{24.8 \%}$ & $\underline{44.5 \%}$ & $\underline{24.8 \%}$ \\
\hline
\end{tabular}

*) Percentage of viable amoebae in comparison to specimens count in control cultures assumed as $100 \%$.

Level of statistical significance set at $\mathrm{p}<0.05$. Statistically significant differences in relation to data regarding cultures not exposed to chemicals are underlined.

Anti- amoebic activity on the corneal Acanthamoeba strain was also revealed in tests with toyocamycin. The statistically significant reduction of the pathogenic amoeba population density was more intense in cultures exposed to Toyo in the concentration of $50 \mu \mathrm{M} / \mathrm{ml}$ than that of $25 \mu \mathrm{M} / \mathrm{ml}$. Simultaneously, the decrease in the number of live amoebae was observed earlier after exposure to Toyo for $48 \mathrm{~h}$ and $96 \mathrm{~h}$, in comparison to the $120 \mathrm{~h}$ observed in cultures exposed to CXD. However, a variable intensity of amoebostatic impact of Toyo on the amoebic populations was revealed during subsequent monitoring days (Tab. 1). Contrary to this observation, antiamoebic activity of PI resulted in a consistent downward trend in corneal Acanthamoeba strain population density.

Protists of the A. castellanii Neff strain indicated more variable sensitivity to the tested agents than that of the corneal Acanthamoeba strain (Tab. 2); this was particularly significant in assays with PI and Toyo. The amoebostatic effect of the iodophore was expressed in a significantly reduced percentage of viable amoebae after120h exposure, up to $14 \%$ in comparison to specimens counts in control cultures assumed as $100 \%$. This effect of PI was more intense than the impact of CXD - 30.8\%, $(\mathrm{p}<0.05)$. In contrast, the amoebae of the strain were clearly more resistant to Toyo than to $\mathrm{CXD}$, e.g. the percentage of live amoebae at $96 \mathrm{~h}$ exposure to this chemical decreased to the level $62.5 \%$, in comparison to $18.2 \%$, respectively $(\mathrm{p}<0.05)$.

Table 2. In vitro effect of tested agents on Acanthamoeba castellanii Neff strain

\begin{tabular}{lccccccc}
\hline \multicolumn{6}{c}{ Live amoebae after exposure to the agents* by the following hours: } \\
\hline Agent & $\begin{array}{c}\text { Concen- } \\
\text { tration }\end{array}$ & $24 \mathrm{~h}$ & $48 \mathrm{~h}$ & $96 \mathrm{~h}$ & $120 \mathrm{~h}$ & $144 \mathrm{~h}$ \\
\hline Povidone iodine & $0.5 \%$ & $\underline{63.7 \%}$ & $\underline{33 \%}$ & $\underline{18.2 \%}$ & $\underline{14 \%}$ & $\underline{14.3} \%$ \\
\hline $\begin{array}{l}\text { Chlorhexidine. } \\
\text { digluconate }\end{array}$ & $0,02 \%$ & $84 \%$ & $67.8 \%$ & $\underline{39.6 \%}$ & $\underline{30.8 \%}$ & $\underline{31 \%}$ \\
\hline \begin{tabular}{l} 
Toyocamycin \\
\cline { 2 - 7 }
\end{tabular} & $25 \mu \mathrm{M}$ & $97.7 \%$ & $\underline{77.83 \%}$ & $\underline{73.50 \%}$ & $89.7 \%$ & $96.24 \%$ \\
\hline
\end{tabular}

* Percentage of viable amoebae in comparison to specimens count in control cultures assumed as $100 \%$.

Level of statistical significance set at $p<0.05$. Statistically significant differences in relation to data regarding cultures not exposed to chemicals are underlined.
In vitro sensitivity of $A$. polyphaga T4 corneal strain and A. castellanii Neff environmental strain to the tested agents is presented in Tables 1 and 2 .

\section{DISCUSSION}

Acanthamoeba keratitis is an emerging, sight-threatening eye disease caused by the facultative parasites of the genus Acanthamoeba, which are ubiquitous in human environments. Without adequate therapy, the amoebic infection that causes a significant deterioration of visual acuity, may lead to blindness.

The knowledge and awareness of vision-threatening Acanthamoebic keratitis as a serious corneal disease are still insufficient, and during the last few decades the cases of AK have been constantly increasing worldwide $[6-9,13$, $15-17,23-25,30]$.

The leading risk factor for $\mathrm{AK}$ is contact lens wear; a corneal epithelial injury and exposure of the eye to water containing cysts and trophozoites of amphizoic amoebae, apart from contact lens wear, are other factors predisposing to AK [8-10, $15-17,30]$.

Diagnostic errors that delay appropriate treatment may result in a prolonged, severe course of $\mathrm{AK}$. It is emphasized in the literature and confirmed by our experience [13-17, 28,30 ] that the eye disease caused by the Acanthamoeba protozoans is often misdiagnosed due to unspecific clinical symptoms, and then unsuccessfully treated as a viral infection with Herpes simplex, bacterial keratitis caused by Pseudomonas aeruginosa or fungal infection caused by Fusarium spp. or Candida spp. Moreover, the mixed keratitis caused by amoebae and concomitant bacterial, viral, or fungal infections may also occur. Non-invasive methods are recommended for preliminary rapid diagnostics, e.g. using the slit lamp for disclosure of an active epithelial inflammation and a visualization of hyper-reflective objects, e.g. cysts of Acanthamoeba by in vivo confocal microscope $[35,36,37]$.

Laboratory microbiology and parasitology examinations of corneal scraping samples by light microscopy are applied for the detection and determination of Acanthamoeba species, and the culture methods are considered the gold standard for the verification/confirmation of the diagnosis $[15,16,17,28,30$, 35-42]. For years, the diagnostics were based on the structure of the amoebic developmental forms. Three morphological groups of species were established within the Acanthamoeba genus based on morphological criteria, mainly cyst size and the number of arm-like structures within a single cyst [14, $15,16,17,30]$. However, pathogenic Acanthamoeba may belong to species similar morphologically, i.e. variation in the cyst morphology occur in A. mauritaniensis, A. castellanii, $A$. polyphaga, and $A$. hatchetii, and additional isolates may be revealed.

Recently, with the development of molecular techniques, the classification of clinical and environmental strains is based on associations of $18 \mathrm{~S}$ rRNA gene sequences, PCR products analysis and sequencing. Obtained sequences are compared with data available in GenBank to determine genotypes of the individual isolates [14-17, 26, 27, 29, 33]; to-date, $18 / 19$ genotypes have been determined $[15,17,30]$.

Apart from misdiagnosis, many factors complicate effective pharmacotherapy in Acanthamoeba keratitis. Different 
viability of amoebic strains, their various susceptibility/ resistance to chemicals, unequal impact of drugs on trophozoites and cysts, various responses of amoebae to formerly effective pharmaceuticals, and the toxicity of chemicals to human corneal cells in effective concentrations result in treatment difficulties. For this reason, the search for substances with possible activity against Acanthamoeba strains is still necessary.

Previous studies by the authors of the current study have shown that cultivation and in vitro monitoring of population dynamics of amoebic strains isolated from the infected corneas may be useful tools for assessment of the viability and changes in morpho-physiological status of the etiological agent of $\mathrm{AK}$, and for relating the data to a prognosis of pharmaceutical efficacy in the disease $[28,30]$. In the presented study, the amoebae of corneal A. polyphaga T4 genotype derived from an eye with a clinically severe course of AK, complicated diagnostically and therapeutically, was assessed. A strong in vitro viability of the amoebae was expressed in their long surviving time linked with the ability of trophozoites to multiply in the axenical culture medium. Moreover, during in vitro monitoring of amoebic population dynamics, significant differences were revealed in resistance/ sensitivity of the corneal strain to the agents tested.

At present, no single chemical has been described as an effective treatment against AK. The selection of drugs to be used and the duration of AK therapy may depend on the

diagnosis time-point, drug resistance of the isolated Acanthamoeba strain, severity of infection related to the amoebic strain viability, and the prevalence of other infectious microorganisms. It has been found that pathogenic isolates may differ in susceptibility to chemical agents/drugs; it is also known that isolates within the same genotype may differ in susceptibility to chemicals $[15,17,26,30,40]$.

The treatment of AK has not yet been fully established. Combination drug therapy is used more or less successfully and mainly involves the topical application of chlorhexidine digluconate, propamidine isethionate (Brolene) and polyhexamethylene biguanide (PHMB), with an addition of antibiotics, such as neomycin or chloramphenicol, to avoid possible bacterial infections $[15,22,43]$. Results of applied therapy are often disappointing due to the resistance of amoebae to disinfectants and drugs. It is emphasized that the extremely high resistance of double-walled Acanthamoeba cysts to chemicals, anti-microbial and anti-parasitic drugs, is considered one of the key contributors of treatment failure $[15,22,31,38,43]$.

Many chemicals have been tested in vitro for their antiamoebic activity and different

Acanthamoeba strains/ isolates of various pathogenicity assessed for their susceptibility/resistance to disinfectants, antiseptics and drugs, including the presented study with nanoparticles as novel therapeutic agents [15, 17, 39-46].

Povidone iodine solution is an agent with broad antibacterial and antiviral activity applied in wound care treatment and preoperative antisepsis. Gatti at al. [40] in their study undertaken in terms of in vitro effectiveness of PI against Acanthamoeba strains, including A. polyphaga, showed some sensitivity of particular amoeba strains to the agent in a solution of $0.5-2.5 \%$, depending on the disinfectant concentrations and type of medium applied for dilutions. The study showed that the PI system is an effective disinfection method for contact lenses. Currently, it is recommended as a contact lens care disinfectant, and also for use as eye drops in some ophthalmic diseases [40-42].

In the current study, a clear, statistically significant amoebostatic effect of povidone iodine has been revealed on both Acanthamoeba strains.

Chlorhexidine digluconate, a cationic antiseptic utilized in prevention and surgeries is currently used in the AK therapy and applied in a concentration of $0.02 \%$. It inhibits membrane function and has been shown previously to have a good in vitro anti-Acanthamoeba activity [15, 17, 40, 43]. However, in spite of the overall amoebicidal in vitro effects, a significant increase in the amoeba cysts level up to $11.6 \%$ was also observed at higher concentrations of the agent, as well as the AK chemotherapeutic resistance or non-responsiveness to CXD [17, 30, 42]. CXD showed moderate amoebostatic effect on the eye amoebic strain.

In the presented study, $0.02 \%$ chlorhexidine activity was also tested against two Acanthamoeba strains. The obtained results were taken as a reference, and the effects of povidone iodine and toyocamycin on the amoebae were compared to those obtained for chlorhexidine digluconate.

Toyocamycin is the adenosine analogue derived from Streptomyces toyocaensis; it blocks the RNA synthesis and ribosome function. A criterion selection of the agent for the current study was its confirmed anti-protozoan activity against Trichomonas sp. and Toxoplasma sp. [43].

To the best knowledge of the authors of the presented study - apart from their preliminary research [44] - this is the first such study undertaken in Poland with diagnosed, pathogenic Acanthamoeba strains leading AK in terms of their in vitro susceptibility to the anti-protozoan agent toyocamycin.

\section{CONCLUSION}

The presented study examined and assessed the anti-amoebic in vitro effects of selected chemicals on the etiological agent of sight-threatening eye disease, Acanthamoebic keratitis, and the influence of the agents on non-pathogenic environmental A.castellanii Neff T4 genotype. It revealed time-dependent amoebostatic in vitro influence of all agents on the Acanthamoeba strains investigated with various degrees of effectiveness. Comparative assessment of the in vitro cultivated Acanthamoeba strains after exposure to the chemicals showed changes in the morpho-physiological status of the populations and number of trophozoites and cysts, as well as the proportion between developmental forms in comparison to the respective control cultures.

The results of the study also revealed that povidone iodine has a better anti-amoebic activity on trophozoites of Acanthamoeba spp. than CXD. Toyocamycin, chemicals with confirmed anti-protozoan effectiveness, was tested in terms of their potential in vitro activity against the Acanthamoeba strains. The agent also indicated that an anti- amoebic effect was reached faster than in samples with CXD; however, this impact was weaker than the influence of IP.

In conclusion, it is noteworthy that none of the tested chemicals in applied concentration induced encystations; simultaneously, no cysticidal effects were caused by these compounds. However, it should be taken into consideration that prolonged in vivo treatment may induce encystations, an undesirable process predisposing to disease recurrence. For this reason, further in vitro investigations on various 
species/strains/isolates of Acanthamoeba, etiological agents of the sight-threatening AK are still necessary. In particular, complementary research should be conducted with different chemicals, modified agent concentrations and application methods in terms of their potential in vitro amoebicidal / cysticidal efficacy, to determine the next possibility for the effective treatment of AK.

\section{REFERENCES}

1. Visvesvara GS, Stehr-Green JK. Epidemiology of free-living ameba infections. J. Protozool. 1990; 37: 25S-33S.

2. Kilvington S, White DG. Acanthamoeba: biology, ecology, and human disease. Rev Med Microbiol. 1990; 5: 12-20.

3. Martinez AJ, Visvesvara GS. Free-living, amphizoic and opportunistic amebas. Brain Pathol. 1997; 7: 583-598.

4. Schuster FL, Visvesvara GS. Free-living amoebae as opportunistic and non-opportunistic pathogens of humans and animals. Int J Parasitol. 2004; 34: 1001-1027.

5. Befinger M, Myjak P, Pietkiewicz H. Occurrence of amphizoic amoebae in lake Żarnowieckie. Bull Inst Mar Trop Med Gdynia. 1986; 37: 275 284.

6. Marciano-Cabral F, Cabral G. Acanthamoeba spp. as agents of disease in humans. Clin Microbiol Rev. 2003; 16: 273-307.

7. Tsvetkova N, Schild M, Panaiotov S, Kurdova-Mintcheva R, Gottstein B, Walochnik J, Aspöck H, Siles Lucas M, Müller N. The identification of free-living environmental isolates of amoebae from Bulgaria. Parasitol Res. 2004; 92: 405-413.

8. Khan NA. Acanthamoeba: biology and increasing importance in human health. FEMS Microbiol Rev. 2006; 30: 564-595.

9. Lorenzo-Morales J, Monteverde-Miranda CA, Jiménez C, Tejedor ML, Valladares BORA. Evaluation of Acanthamoeba isolates from environmental sources in Tenerife, Canary Islands, Spain. Ann Agric Env Med. 2005; 12: 233-236.

10. Wesołowska M, Cisowska A, Myjak P, Marek J, Jurowska-Liput J, Jakubaszko J. Acanthamoeba keratitis in contact lens wearers in Poland. Adv Clin Exp Med.2006.; 15: 553-555.

11. Łanocha N, Kosik-Bogacka D, Maciejewska A, Sawczuk M, Wilk A, Kuźna-Grygiel W. The occurrence Acanthamoeba (free living amoeba) in environmental and respiratory samples in Poland. Acta Prot. 2009; 48: 271-279.

12. Trabelsi H, Sellami A, Dendena F, Sellami H, Cheikh-rouhou F, Makni F, Makni F, Ben DS, Ayadi A. Free-living amoebae (FLA): morphological and molecular identification of Acanthamoeba in dental unit water. Amibes libres: identification morphologiqueet moléculaire d'Acanthamoeba dans l'eau des unités dentaires. Parasite. 2010; 17: 67-70.

13. Walochnik J, Scheikl U, Haller-Schober EM. Twenty years of Acanthamoeba diagnostics in Austria. J Eukaryot Microbiol. 2015, 62: 3- 11. https://doi.org/10.1111/jeu.12149

14. Lass A, Szostakowska B, Idzińska A, Chomicz L. The first genotype determination of Acanthamoeba potential threat to human health, isolated from natural water reservoirs in Poland. J Parasitol Res. 2014; 113: 2693-2699. https://doi.org/10.1007/s00436-014-3925-6

15. Lorenzo-Morales J, Khan NA, WalochnikJ. An update on Acanthamoeba keratitis: diagnosis, pathogenesis and treatment. Parasite. 2015; 22: 1-20. https://doi.org/10.1051/parasite/2015010

16. Chomicz L, Conn DB, Padzik M, Szaflik JP, Walochnik J, Zawadzki PJ, Pawłowski W, Dybicz M. Emerging threats for human health in Poland: pathogenic isolates from drug resistant Acanthamoeba keratitis monitored in terms of their in vitro dynamics and temperature adaptability. BioMed Res Int. Hindawi Publishing Corporation 2015, Article ID 231285, doi.org/ 10.1155/2015/231285.

17. Khan NA. Acanthamoeba: Biology and Pathogenesis. 2nd ed, Caister Academic Press. Norfolk, UK. 2015; 1 - 295.

18. Berger P, Papazian L, Drancourt M, Lascolat B, Auffray JP, Raoult D. Amoeba associated microorganisms and diagnosis of nosocomial pneumonia. Emerg Inf Dis. 2006; 12: 248-255.

19. Červa L, Novak K. Amoebic meningoencephalitis: sixteen fatalities. Science. 1968; 160: 92.

20. Nagington PG, Watson TJ. Amoebic infection of the eye. Lancet. 1974; 304: 1537-1540.

21. Ibrahim YW, Boase DL, Cree IA. Factors affecting the epidemiology of Acanthamoeba keratitis. Ophthalmic Epidemiol. 2007; 14: 53-60.
22. Clarke B, Sinha A, Parmar DN, Sykakis E. Advances in the diagnosis and treatment of Acanthamoeba keratitis. Hindawi. J Ophthalmol. 2012; Article ID 484892, 6 pages. https://doi.org/10.1155/2012/484892

23. Szaflik JP, Padzik M, Chomicz L, Olędzka G, Izdebska J. Przydatność diagnostyki in vitro w trudnych przypadkach Acanthamoeba keratitis, wymagających postępowania farmakoterapeutycznego i chirurgicznego. (Usefulness of in vitro diagnostics in difficult incidences of Acanthamoeba keratitis requiring pharmacotherapy and surgical management.) (in Polish with summary in English). Okulistyka. 2012; 3: 28-32

24. Lorenzo-Morales J, Martín-Navarro CM, López-Arencibia A, ArnalichMontiel F, Piñero JE, Valladares B. Acanthamoeba keratitis: an emerging disease gathering importance worldwide? Trends Parasitol. 2013; 29: 181-187. https://doi.org/10.1016/j.pt.2013.01.006

25. Page MA, William DM. Acanthamoeba Keratitis: A 12-year experience covering a wide spectrum of presentations, diagnoses, and outcomes. J Ophthalm. 2013; 2013: 1-6.

26. Risler A, Coupat-Goutaland B, Pelandakis M. Genotyping and phylogenetic analysis of Acanthamoeba isolates associated with keratitis. Parasitol Res. 2013; 112: 3807-3816. https://doi.org/10.1007/ s00436-013-3572-3

27. Padzik M, Chomicz L, Szaflik JP, Chruscikowska A, Perkowski K, Szaflik J. In vitro effects of selected contact lens care solutions on Acanthamoeba castellanii strains in Poland. Exp Parasitol. 2014; 145: 98-101.

28. Chomicz L, Padzik M, Szaflik JP, Nahorski WL, Kryczka T, Szaflik J. Monitoring of in vitro dynamics of Acanthamoeba strains isolated from infected eyes as a useful tool in keratitis management. Exp Parasitol. 2014; 145: 73-77.

29. Derda M, Solarczyk P, Cholewiński M, Hadaś E. Genotypic characterization of amoeba isolated from Acanthamoeba keratitis in Poland. Parasitol Res. 2015; 114: 1233-1237.

30. Chomicz L, Szaflik JP, Padzik M, Izdebska J. Acanthamoeba keratitis: The emerging vision - threatening corneal disease. Advances in Common Eye Infections. Ed.: Shimon Rumelt. Published by INTECH 2016; 2: 99-120.

31. Aksozek A, McClellan K, Howard K, Niederkorn JY, Alizadeh H. Resistance of Acanthamoeba castellanii cysts to physical, chemical and radiological conditions. J Parasitol. 2002; 88: 621-623.

32. Chomicz L, Padzik M., Graczyk Z., Starosciak B.,Graczyk T., Naprawska A., Olędzka G., Szostakowska B. Acanthamoeba castellanii: in vitro effects of selected biological, physical and chemical factors. Exp Parasitol. 2010; 126: 103-105. doi:10.1016/j.exppara.2010.01.025

33. Schroeder JM, Booton GC, Hay J, Niszl IA, Seal DV, Markus MB, Fuerst PA, Byers TJ. Use of subgenic $18 \mathrm{~S}$ ribosomal DNA PCR and sequencing for genus and genotype identification of Acanthamoebae from humans with keratitis and from sewage sludge. J Clin Microbiol. 2001; 39: 1903-1911.

34. Sharma M, Bloch A, Bobek M. A practical synthesis of the antibiotic toyocamycin. Nucleos Nucleot Nucl. 1993; 12: 643-648.

35. Parmar DN, Awwad ST, Petroll WM., Bowman RW, McCulley JP., Cavanagh HD. Tandem scanning confocal microscopy in the diagnosis of suspected Acanthamoeba keratitis. Ophthalmology 2006; 113: 538547.

36. Szaflik JP. Comparison of in vivo confocal microscopy of human cornea by white light scanning slit and laser scanning systems. Cornea 2007; 26: 438-445. https://doi.org/10.1097/ICO.0b013e31803dcd11.

37. Padzik M, Szaflik JP, Baltaza W, Perkowski K, Dybicz M, Chomicz L. In vivo confocal microscopy and in vitro culture techniques as tools for evaluation of severe Acanthamoeba keratitis incidents. Annals Parasit. 2017; 63: 341-346. doi:10.17420/ap6304.121.

38. Baltaza W, Padzik M, Szaflik JP, Dybicz M, Hendiger E, Chomicz L. Amoebicidal or amoebostatic influence of disinfectants used in health facilities and laboratories on corneal strains of Acanthamoeba. Annals Parasit. 2017; 63: 167-172. https://doi:10.17420/ap6303.102.

39. Padzik M, Hendiger EB, Chomicz L, Grodzik M, Szmidt M, Grobelny J, Lorenzo-Morales J. Tannic acid-modified silver nanoparticles as a novel therapeutic agent against Acanthamoeba. Parasitol Res. 2018; 117: 3519-3525. https://doi.org/10.1007/s00436-018-6049-6

40. Gatti S, Cevini C, Bruno A, Penso G, Rama P, Scaglia M. In vitro effectiveness of povidoneiodine on Acanthamoeba isolates from human cornea. Antimicr Ag Chemother. 1998; 10: 2232-2234.

41. Kilvington S. Antimicrobial efficacy of a povidone-iodine (PI) and a one-step hydrogen peroxide contact lens disinfection system. Contact Lens and Anterior Eye. 2004; 27: 209-212.

42. Martín-Navarro CM, Lorenzo-Morales J, López-Arencibia A, Valladares B, Piñero JE. Acanthamoeba spp.: Efficacy of Bioclen FR 
One Step, a povidone-iodine based system for the disinfection of contact lenses. Exp Parasitol. 2010; 126: 109-112.

43. Seal DV, Hay J, Kirkness CM. Chlorhexidine or polyhexamethylene biguanide for Acanthamoeba keratitis. Lancet 1995; 345: 136.

44. Chomicz L, Padzik M, Piekarczyk J, Graczyk Z, Godineau N. Szałwiński M, Piekarczyk P, Graczyk T, Olędzka G, Kazimierczuk Z. Effects of metronidazole, chlorhexidine digluconate and new-synthesized benzimidazole and nitroimidazole derivatives on in vitro viability of selected protozoans - potential factors of intra and after -surgery complications. Chir. Czaszk Szczęk Twarz Ortop Szczęk. 2008; III, 6: $168-174$.
45. Wright JM, Dunn LA, Kazimierczuk Z, Burgess AG, Krauer KG, Upcroft P, Upcroft JA. Susceptibility in vitro of clinically metronidazoleresistant Trichomonas vaginalis to nitazoxanide, toyocamycin, and 2-fluoro-2'-deoxyadenosine. Parasitol Res. 2010; 107: 847-853.

46. Padzik M, Szaflik JP, Izdebska J, Szostakowska B, Szaflik J, Chomicz L. Acanthamoeba polyphaga strain - causative agent of vision-threatening keratitis uncommonly detected in Poland, susceptible in vitro to toyocamycin. Klinika Oczna. 2017; 119: 77-82. ISSN 0023-2157 Index 362646 